\title{
Erwiderung auf vorstehende Bemerkung ${ }^{1}$. \\ Von
}

Prof. Dr. Neisser-Stettin.

Die vorstehende Bemerkung der Herren Löwenstein und Rappoport ändert nicht das geringste an dem, was ich in meinem Referat, sowie in meinem Aufsatz im vorigen Heft dieser Beiträge über ihre Schlusstabelle gesagt habe.

1) Am 26. November habe ich in der zweiten Versammlung der Tuberkuloseärzte ein Referat: „Weitere Erfahrungen über Tuberkulin-Anwendung in Heilstätten $^{\prime \prime}$ gehalten. Whe dasselbe im Druck erschienen war - auf Grund des vorläufigen Referats vom Kollegen $\mathrm{Köhler-Holsterhausen} \mathrm{-} \mathrm{erschien} \mathrm{ein} \mathrm{Artikel}$ der Herren Löwenstein und Rappoport in der Zeitsehrift für Tuberkulose und Heilstättenwesen, Bd. 6, Heft 6, 1905: „Bemerkung zu dem von Herrn Dr. $\mathrm{N}_{\text {e }}$ s s e r - Stettin in der Versammlung der Tuberkuloseärzte in Berlin am 26. November 1904 erstatteten Referate über Tuberkulinbehandlung" nebst einer Anmerkung von Herrn Möller, deren Charakterisierung ich an anderem Orte bereits gegeben habe. Dieser Angriff wurde in der Zeitschrift für Tuberknlose und Heilstättenwesen gedruckt, obne dass mir davon Kenntnis bezw. Gelegenheit gegeben wurde, in der gleichen Nummer der Zeitschriftza ant. worten.

Ich wandte mich deshalb an die Redaktion der „Beiträge zur Klinik der Tuberkulose" mit der Bitte, um Aufnahme meiner Abwehr: „Die Schlusstabelle in der Arbeit ${ }_{n}$ Über den Mechanismus der Tuberkulinimmunität ${ }^{*}$ von E. Lörenstein und E. Rappoport."

Diese Abwehr sandte ich am 8. März vor ihrem Erscheinen an Herrn von Leyden nebst Begleitschreiben, in welchem ich Herrn von Leyden auf das Vorgehen der Zeitschrift für Tuberkulose, sowie auf die Anmerkung von Herrn Möller aufmerksam zu machen mir erlaubte.

Nachdem ich am 16. März ron Herrn von Leyden brieflichen Bescheid erhalten hatte, erhielt ich am 18. März von der Redaktion der Zeitschrift für Tuberkulose den Entwurf der obigen Bemerkung der Herren Lö wenstein und $R$ a p poport zugesandt (ohne den Nachtrag) mit der Frage, ob ich in der Zeitschrift für Tuberkulose zu antworten wünschte. Hierauf schickte ich meine erste Abwehr direkt an die Redaktion der Zeitschrift für Tuberkulose zur Kenntnisnahme für die Herren Löwenstein und Rappoport und erhielt nun den Bescheid, dass die Herren nicht in der Zeitschrift für Tuberkulose, sondern ebenfalls in den „Beiträgen“ antworten würden. 
In ibrem ersten Angriff auf mein Referat haben die Herren Autoren versucht, meine Kritik ihrer Schlusstabelle dadurch abzuwehren, dass sie sagten, für die von ibnen publizierte Tabelle wäre die auf Seite 493 ihrer Arbeit genauest angegebene Einteilung massgebend gewesen.

Dem gegenüber habe ich in meinem oben genannten Artikel nachgewiesen, dass die dortstehende Einteilung (eine 2-bezw. 5-Teilung) nämlich :

$$
\left.\begin{array}{l}
\text { A I, II geschlossene } \\
\text { B I, II, III offene }
\end{array}\right\} \text { Tuberkulose }
$$

weder in der Schlusstabelle selbst angewendet ist, noch dass irgendwie in der Arbeit bemerkt oder erkennbar gemacht ist, dass oder in welcher Weise aus dieser 2- bezw. 5-Teilung eine 3-Teilung gemacht worden ist, noch dass es etwa möglich ist, aus den „zur Kontrolle“ vollständig publizierten Krankenprotokollen, auf denen die Angaben der Autoren basieren sollen, zu den Zahlen ihrer Schlusstabelle zu gelangen.

In der jetzigen Bemerkung der Autoren ist denn auch keine Rede mehr davon, dass, wie in der ersten Bemerkung behauptet, die Einteilung A I, II, B I, II, III der Schlusstabelle zugrunde läge. Es wird vielmehr jetzt gesagt: "Wir haben die für die Behandlung mit Tuberkulin geeigneten Fälle allein in drei Stadien eingeteilt - so dass keine Rede davon sein kann, wie der Herr Referent es getan hat unsere Stadieneinteilung mit der Möllerschen ïblichen zu identifizieren."

Hierauf erwidere ich am besten, indem ich das zitiere, was ich auf. Seite 258 meines ersten Artikels gesagt habe:

„Sehen wir uns den Schluss der Löwenstein- und Rappoportschen Arbeit an, so bèisst es dort: Beim Vergleiche der beiden folgenden Tabellen wird man sich der Ansicht wohl nicht verschliessen können, dass dem Tuberkulin bei der Behandlung der Tuberkulose eine wesentliche Rolle zuzuschreiben ist."

Von diesen Tabellen ist die untere (Hyg.-diät. Behandlung), wie leicht festzustellen ist, aus dem Mölle r schen 3. Jahresbericht zifferngetreu übernommen. Es handelt sich also - was aber die Autoren in der Arbeit keineswegs sagen, was vielmehr nur durch Studium des Jahresberichtes und Ausrechnung der Prozentzahlen sich ergibt um die Heilresultate der hygienisch-diätetischen Behandlung der Jahre 1901 und 1902 und die angewendete Einteilung I, II, III bedeutet die üblichen Stadien der Tuberkulose (Turban-Möller).

Folglich wird jedermann als selbstverständlich annehmen, dass auch in der zum Vergleiche daneben" gestellten oberen Tabelle (kom- 
binierte Behandlung) die Ziffern I, II, III dieselbe Bedeutung haben und ebenfalls die üblichen Stadien der Tuberkulose bedeuten sollen.".

Wenn das also nicht der Fall ist und Stadium I, II, III in Tabelle I etwas ganz anderes bedeutet als in der anderen Tabelle - und zwar ohne dass dies im geringsten erkennbar gemacht ist,

Kombinierte Behandlung.

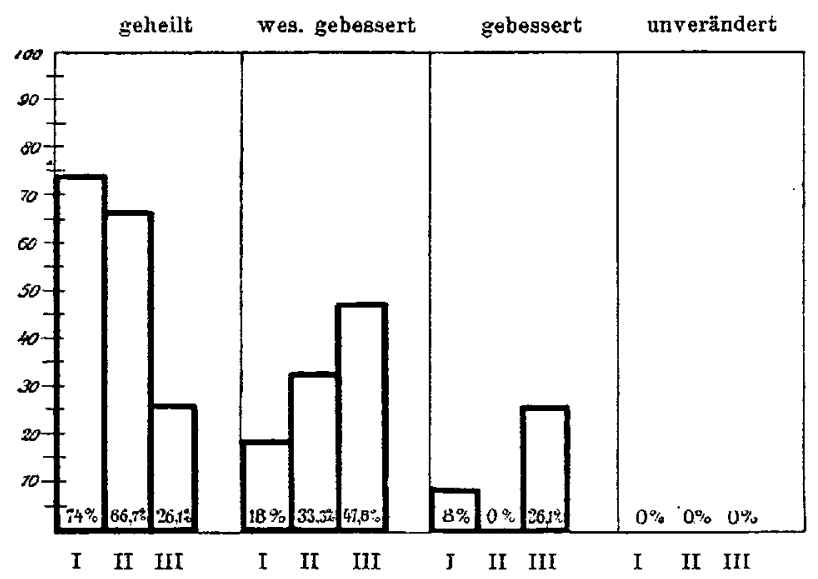

Hygienisch-diätetische Behandlung.

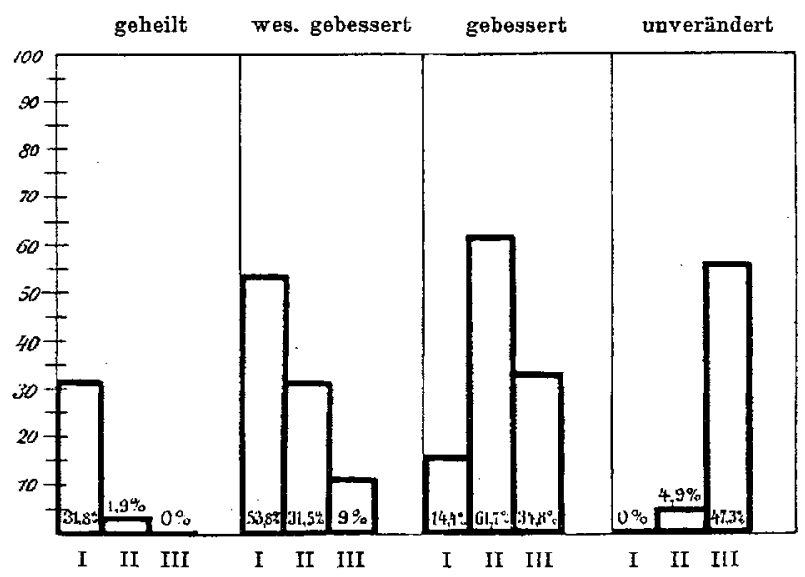

so wird hierdurch in gänzlich unzulässiger Weise zu einem Vergleiche aufgefordert, und ein falsches Bild von der Tuberkulinheilwirkung erzeugt. -

Wie? Glauben die Autoren nun wirklich den Eindruck dieser Ausführungen dadurch abschwächen zu können, dass sie anstatt der 
von mir referierten Arbeit eine andere von mir nicht referierte Arbeit vorschieben?

In dieser andern, von mir nicht referierten Arbeit ist - das soll hier schärfstens hervorgehoben sein - nämlich keineswegs der von mir getadelte Versuch gemacht, einen Vergleich der kombiniert behandelten 85 Fälle nach Heilprozenten mit den 602 hyg.diätetisch behandelten aus dem Möllerschen Jahresbericht in der von mir beanstandeten Weise anzustellen.

Ein kleines Beispiel wird vollkommen genügen.

In der von mir nicht referierten und nicht beanstandeten Arbeit heisst es ganz klar: Von 21 schweren offenen Tuberkulosen wesentlich gebessert 9 Fälle.

In der von mir referierten und beanstandeten Arbeit stehen dagegen keine Zahlen sondern:

\begin{tabular}{cccccc}
\multicolumn{2}{c}{ Kombinierte Behandlung } & \multicolumn{3}{c}{ Hyg.-diät. Behandlung } \\
I II & III & I & II & III \\
wesentlich & & & & \\
rert wurden: & $47,8 \%$ & & & $9 \%$
\end{tabular}

gebessert wurden: $\quad 47,8 \% \quad 9 \%$

wobei wohlgemerkt in nicht erkennbar gemachter Weise III links ganz etwas anderes bedeutet als III rechts. Es wird also jedermann den Eindruck erhalten, dass man - gleichviel ob ein Abzug ron nicht geeigneten Fällen stattgefunden hat - aus der Tabelle schliessen soll, dass bei Studium III durch die kombinierte Behandlung ganz andere Erfolge erzielt worden seien, als durch die hyg.-diätetische Behandlung.

Aber weiter. Die Autoren sagen oben: „Dementsprechend kamen von 189 mit Alt-Tuberkulin behandelten Fällen nur 85 in Betracht, welche obige Bedingung erfüllten."

Diese Zahlen stehen nicht etwa in der von mir referierten Arbeit, vielmehr sind sie wieder aus der anderen Arbeit entnommen. Aber vielleicht ist wenigstens - um dies an sich unberechtigte Vorschieben einer anderen Arbeit seitens der Autoren einigermassen zu rechtfertigen - das Material beider Arbeiten dasselbe?

Das ist, wie man sich durch Nachrechnen leicht überzeugen kann, nicht der Fall.

Wir geben folgendes Beispiel: 


\begin{tabular}{l||c|c}
\hline \hline & Referierte Arbeit & Andere Arbeit \\
\hline \hline Gesamtzahl der Fälle. . . . . . . & 176 & 189 \\
davon geschlossene Tuberkulose (in Be- & 18 & 48 \\
tracht kommende Fälle). . . . . & 12 & 35 \\
davon geheilt. . . . . . . . . & 12 &
\end{tabular}

In der referierten Arbeit der Autoren sind weder die genannten Zahlen, noch überhaupt die für die Schlusstabelle benützten Zahlen angegeben oder kenntlich gemacht - und das ist sehr erklärlich denn - wie schon mehrfach ausgeführt - ist es entgegen ihren Angaben unmöglich auf Grund des Materials, das die Autoren in extenso geben, zur Schlusstabelle zu gelangen. Und doch erklären sie ausdrücklich :

"Wir haben uns die Mühe nicht verdriessen lassen, unsere vollständigen Krankenprotokolle zu publizieren, auf welchen unsere Angaben basieren, von dem Wunsche geleitet, den Gegnern der Tuberkulintherapie eine Kontrolle über unsere Beobachtungen in die Hand zu geben."

Die Hineinziehung der anderen Arbeit und die „klare zahlenmässige Ausführung“ der Autoren, entfernt davon unsere früheren Ausführugen zu widerlegen, lässt die Herstellung ihrerSchlusstabelle in nur um so weniger günstigem Lichte erscheinen.

In der obigen Bemerkung wenden sich die Autoren noch gegen eine andere kritische Bemerkung meines Referates, des Inhaltes, dass gegenüber der Angabe Möllers, dass in der Regel frisch Erkrankte auf kleine Dosen hoch reagieren, es in Erstaunen setzen werde, dass die Herren Autoren am Belziger Material zu dem Resultat kommen, dass mit dem Fortschreiten des Krankheitsprozesses die Grösse der minimalen Reaktionsdosis sinkt, dass Leichtkranke in der Regel auf eine höhere, Schwerkranke auf eine niedere Dosis reagieren.

Die Herren Autoren nehmen an, dass hier ein Irrtum meinerseits vorliege, insofern als ich "Früherkrankung und Leichterkrankung obne weiteres identifiziere“.

Das ist keineswegs der Fall, wohl aber bin ich der Meinung and werde damit kaum , allein unter den Tuberkuloseärzten dastehen ${ }^{*}$ - dass unter den frischen Fällen in der Regel die weniger ausgedehnten Prozesse, die Anfangsstadien, unter den chronischen Fällen 
die fortgeschrittenen Prozesse $\mathrm{zu}$ finden sind. Oder - wozu treiben wir sonst Frühdiagnostik?

Demnach wird man sich - genau so wie ich das im Referat zum Ausdruck gebracht habe, billig wundern dürfen, dass nach Möller die frisch Erkrankten, unter denen sich doch vorzugsweise die Anfangsstadien befinden, schon auf kleine Dosen reagieren, während nach den Autoren gerade die fortgeschrittenen Fälle im Gegensatz zu den Anfangsstadien auf kleine Dosen hoch reagieren sollen.

Nun zum Schlusspassus der Autoren.

An der hier zitierten Stelle meines Referats habe ich davor gewarnt, etwa aus der von den Herrn Autoren gefundenen Tatsache, dass die Anfangsstadien der Tuberkulose eine höhere minimale Reaktionsdosis als die fortgeschritteneren Fälle haben sollen, den naheliegenden Schluss zu ziehen, dass man diesen Fällen von vornherein mit grösseren Tuberkulindosen za Leibe rücken dürfe. Man solle vielmehr zweckmässigerweise die Eigenschaft dieser Fälle benützen, dass sie auf mehrfach wiederholte kleine Dosen besonders empfindlich werden. Ich führte ausdrücklich im Referat an, dass diese Eigenschaft der genannten Fälle „auch von den Autoren betont wird“, es ist also unbegreiflich, wie von den Autoren hieraus auf eine mangelhafte Lektüre ihrer Arbeit meinerseits geschlossen werden kann. Im Gegenteil mussten sie daraus schliessen, dass ich aus ihrer 50 Seiten langen Arbeit die einzig hierher gehörige Stelle auf Seite 497 genau gekannt habe.

Bei dieser Sachlage muss ich es als ein starkes Stück bezeichnen, wenn die Autoren auf Grund einer so gänzlich haltlosen Angabe dem Referenten über einige 20 Tuberkulinarbeiten öffentlich ungenügende Information über ihre Arbeit vorwerfen.

Stettin, 22. März 1905.

\section{Druckfehler-Berichtigung.}

Aaf Seite 322,15 . u. 16. Zeile ron unten lies $\operatorname{Seh} w a n k u n g \theta n$ anstatt Schwäehungen. 